\title{
General overview of black hole accretion theory
}

\author{
Omer Blaes
}

Received: date / Accepted: date

\begin{abstract}
I provide a broad overview of the basic theoretical paradigms of black hole accretion flows. Models that make contact with observations continue to be mostly based on the four decade old alpha stress prescription of Shakura \& Sunyaev (1973), and I discuss the properties of both radiatively efficient and inefficient models, including their local properties, their expected stability to secular perturbations, and how they might be tied together in global flow geometries. The alpha stress is a prescription for turbulence, for which the only existing plausible candidate is that which develops from the magnetorotational instability (MRI). I therefore also review what is currently known about the local properties of such turbulence, and the physical issues that have been elucidated and that remain uncertain that are relevant for the various alpha-based black hole accretion flow models.
\end{abstract}

Keywords Accretion, accretion disks · black hole physics · instabilities · MHD

\section{Introduction}

Accretion is the very process that allows black hole sources to emit electromagnetic radiation and other forms of energy. Because black holes are so small in size compared to the spatial scale of their sources of fueling, and because centrifugal forces on matter of given angular momentum increase more rapidly $\left(\propto R^{-3}\right)$ than gravity $\left(\propto R^{-2}\right)$ as one moves inward in radius $R$, accretion is generally believed to be a process involving rotationally supported flows. Matter in such a flow must lose angular momentum in order to move inward and release gravitational binding energy. It is the nature of the angular momentum loss mechanism, and the process whereby gravitational binding energy is converted into observable forms of energy, that are the two central questions of black hole accretion theory. At least three mechanisms have been proposed for angular momentum extraction:

O. Blaes

Department of Physics, University of California, Santa Barbara, CA 93106, USA

Tel.: +1-805-8937239

E-mail: blaes@physics.ucsb.edu 
(1) External stresses associated with large scale magnetic fields in a magnetohydrodynamic (MHD) outflow. This mechanism (Blandford \& Pavne 1982) may be relevant in low luminosity sources where accretion power may be largely converted into mechanical power in outflows. It may also be relevant in resolving the fueling and self-gravity problems in the outer accretion flows in active galactic nuclei (Goodman 2003). Whether and how large scale magnetic fields can be created remains an open question, however.

(2) Magnetorotational (MRI) turbulence. Such turbulence is generic for plasmas that are sufficiently electrically conducting and not too strongly magnetized (Balbus \& Hawley 1991; Hawlev \& Balbus 1991; Balbus \& Hawlev 1992, 1998). Because turbulence is inherently dissipative, this process is almost certainly relevant for sources whose power output is dominated by thermal radiative emission mechanisms.

(3) Nonaxisymmetric waves and shocks. Nonaxisymmetric (e.g. spiral) waves can transport angular momentum outward through the flow. Such waves can also transport energy away from the region where gravitational binding energy is released, depositing it elsewhere. Waves are almost certainly relevant in disks around supermassive black hole binaries, and also in the outer, self-gravitating parts of disks in active galactic nuclei. They probably also play a role in the outer parts of black hole X-ray binary disks due to tidal excitation by the companion star. Nonaxisymmetric shocks can also play an important role in the inner regions of accretion flows whose angular momenta are misaligned with the black hole spin axis (Fragile \& Blaes 2008).

Among these options, only the second - MRI turbulence - is a mechanism that might be describable by the classical alpha prescription of Shakura \& Sunvaev (1973), at least in some aspects (Balbus \& Papaloizou 1999). The angular momentum transporting stress $w_{R \phi}$ in the turbulence is given by local space and time averages of correlated fluctuations in radial $(R)$ and azimuthal $(\phi)$ fluctuations of velocity $\mathbf{v}$ (the Reynolds stress) and magnetic field B (the Maxwell stress),

$$
w_{R \phi}=\left\langle\rho v_{R} \delta v_{\phi}-\frac{B_{R} B_{\phi}}{4 \pi}\right\rangle,
$$

where $\rho$ here is the mass density and $\delta v_{\phi}$ is the local deviation of the azimuthal velocity component from the mean background shear flow. Maxwell stresses are generally larger in magnitude than the Reynolds stresses by factors of at least several. I say that the total stress might be describable by the classical alpha prescription because these stresses appear to be mostly local in the sense that simulations show that radial correlations in stress drop rapidly on scales larger than the local disk scale height. However, as I discuss in section 3.1 below, there remain correlations on larger radial scales.

This article provides a broad overview of alpha-based models of black hole accretion flows, focusing on structure, dynamics, and thermodynamics. These models continue to dominate theoretical efforts to explain observations, but a slow revolution is occurring as simulations of MRI turbulence, both local and global, continue to become more powerful and to incorporate more and more of the relevant physics. This article will also discuss what has been learned recently from local, shearing box simulations of MRI turbulence as this pertains directly to some of the alpha-based modeling. A review of global simulations can be found in Chapter 2.4. Spectral modeling of accretion flows is discussed in Chapter 2.3. I will also mainly focus on accretion rather than the 
formation of jets and outflows here, though jets and outflows are clearly important (both observationally and theoretically, in certain flow states). See Chapter 5.3 on jet launching mechanisms.

\section{Hydrodynamic disk models with the alpha prescription}

Decades of theory and models of black hole accretion flows have critically relied on the alpha prescription for a local stress introduced by Shakura \& Sunvaev (1973). There are numerous variants of this prescription which produce order unity changes in the definition of $\alpha$, and one of the most common is

$$
w_{R \phi}=\alpha P,
$$

where $P$ is the thermal pressure. Most models have assumed that this is the total thermal pressure (gas plus radiation), but prescriptions in which the stress is taken to be proportional to just the gas pressure alone (e.g. Sakimoto \& Coroniti 1981) or the geometric mean of the gas and total thermal pressures (e.g. Taam \& Lin 1984) have also been suggested. However, as illustrated in Figure 1 recent radiation MHD simulations of MRI turbulence find that the stress scales best with total thermal pressure, at least on long time scales (Ohsuga et al. 2009; Hirose. Blaes \& Krolik 2009).

The alpha prescription (2) is usually used to solve for the radial structure of vertically-integrated geometrically thin or slim accretion disks, in which case it enters the equations through the vertically-integrated stress:

$$
W_{R \phi}=\int_{-\infty}^{\infty} w_{R \phi} d z \sim 2 H \alpha P
$$

where $P$ is now some vertically averaged thermal pressure, of order the midplane pressure, and $H$ is the vertical half-thickness of the disk. This is consistent with MRI simulations, but the prescription is also occasionally used even more locally by assuming that the vertical profiles of stress and dissipation at a given radius are proportional to the local vertical profile of thermal pressure. As we discuss below in section 3.2, this is not consistent with vertically stratified simulations of MRI turbulence, which generally have vertical profiles of stress that are broader than the thermal pressure profile. Alpha defined locally would therefore increase rapidly outward from the disk midplane.

\subsection{Local thermal equilibria and secular instabilities}

Virtually all (non-simulation-based) models of black hole accretion flows are based on vertically integrated hydrodynamic equations. These models often neglect the possibility of significant losses of mass, angular momentum, and energy in outflows and jets, though some models do attempt to include them with various prescriptions, particularly in advection-dominated flows which we will come to shortly. As discussed in Chapter 5.1, neglect of outflows is likely to be a bad approximation in some sources and accretion states. Nevertheless, if we adopt this assumption for simplicity, then for stationary flows, the conservation laws of mass, radial momentum, angular momentum, and internal energy can be written as

$$
\dot{M}=2 \pi R \Sigma v,
$$




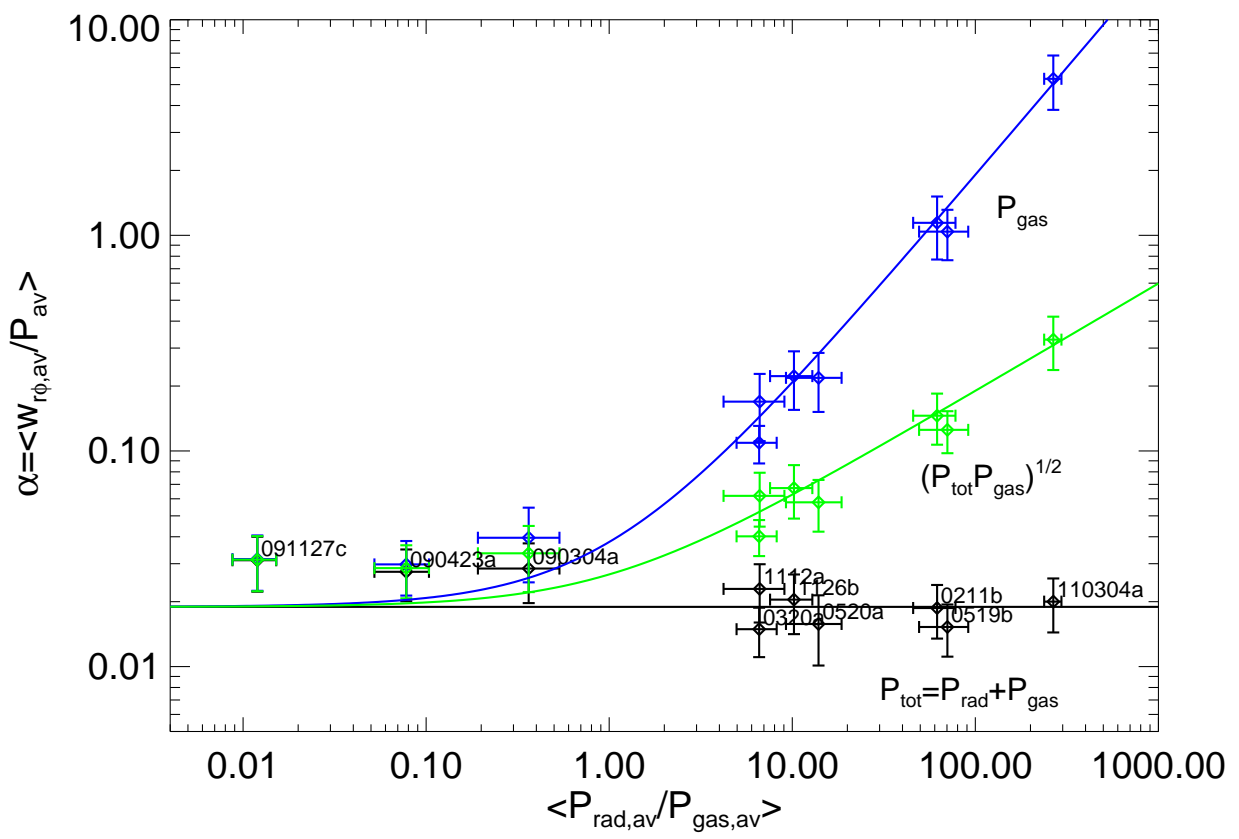

Fig. 1 Time-averaged values of the ratio of spatially averaged stress to various measures of spatially averaged thermal pressure, i.e. the Shakura \& Sunvaev (1973) parameter alpha, as a function of the time-averaged ratio of spatially averaged radiation pressure to spatially averaged gas pressure, for a number of radiation MHD, vertically stratified, shearing box simulations of MRI turbulence. Black, green and blue points are the results for thermal pressures defined to be the total (radiation plus gas) pressure, the geometric mean of the total and gas pressures, and the gas pressure alone, respectively. Horizontal and vertical error bars on all points indicate one standard deviation in the respective time-averages. The horizontal black line is the average alpha value of the total pressure prescription (black) points, while the green and blue curves are what would result if the total pressure prescription were correct, but one nevertheless insisted on defining alpha in terms of the other thermal pressure definitions used in the green and blue points, respectively. The stress prescription that is most consistent with the simulation data is one in which the total thermal pressure is used, though it is perhaps noteworthy that the alpha values in the gas pressure dominated simulations are consistently higher than the alpha values in the radiation pressure dominated simulations. (Updated from Hirose, Blaes \& Krolik 2009.)

$$
\begin{gathered}
\rho v \frac{d v}{d R}=\rho\left(\Omega^{2}-\Omega_{\mathrm{K}}^{2}\right) R-\frac{d P}{d R}, \\
\dot{M} \frac{d \ell}{d R}=\frac{d}{d R}\left(2 \pi R^{2} W_{R \phi}\right),
\end{gathered}
$$

and

$$
Q_{\mathrm{adv}} \equiv \frac{-\dot{M}}{4 \pi R}\left[\frac{d U}{d R}+P \frac{d}{d R}\left(\frac{1}{\rho}\right)\right]=Q^{+}-Q^{-},
$$

where we have neglected general relativity for the purposes of physical transparency. Here $\rho \sim \Sigma /(2 H)$ is a vertically-averaged density, $\Sigma$ is the surface mass density, $\Omega$ is the fluid angular velocity which may differ from the test particle (Kepler) angular velocity $\Omega_{\mathrm{K}}, \ell=\Omega R^{2}$ is the fluid specific angular momentum, $v$ is the inward radial drift speed, $U$ is a vertical average of the internal energy per unit mass, $Q^{-}$is the radiative cooling 
rate per unit surface area on each face of the disk, $Q^{+}=-(1 / 2) W_{R \phi} R d \Omega / d R$ is half the turbulent dissipation rate per unit surface area, and $Q_{\text {adv }}$ is half the inward radial advection of heat per unit surface area. Vertical hydrostatic equilibrium implies that the vertical half-thickness of the disk is $H \sim(P / \rho)^{1 / 2} / \Omega_{\mathrm{K}}$.

Once one adopts the alpha prescription (2), together with an equation of state and opacities and/or optically thin cooling functions, it is possible to solve these equations with assumed boundary conditions to derive the radial profiles of vertically-averaged fluid variables in the flow. Such models generally invoke a regularity condition at an inner sonic point and/or a no-torque inner boundary condition at, for example, the innermost stable circular orbit, although magnetohydrodynamic stresses can be important once one enters the plunging region near the black hole (Gammie 1999; Krolik 1999). (See Chapter 2.4 and, e.g., Penna et al. 2010 and Noble, Krolik \& Hawley 2010 for recent simulation work on this issue for geometrically thin disks.) Another approach is to consider radii much larger than the gravitational radius $R_{\mathrm{g}} \equiv G M / c^{2}$ and invoke self-similarity by assuming a constant ratio of advective cooling over turbulent dissipation $Q_{\text {adv }} / Q^{+}$(Naravan \& Yi 1994).

For a fixed black hole mass, models that are stationary generally depend on a number of chosen parameters, the most important being the accretion rate $\dot{M}$ which is everywhere constant through the flow (remember, we are neglecting outflows here). A number of possible equilibria have been discovered in this way, and the primary method of choosing which ones are physically realizable in nature has been to check if they are stable to secular perturbations. The growth rates of such instabilities are related to one of two characteristic time-scales. The first is the thermal time, defined as the characteristic heating time

$$
t_{\mathrm{th}} \sim \frac{U \Sigma}{2 Q^{+}} \sim \frac{1}{\alpha \Omega} .
$$

Thermal instabilities, in which a local patch of the flow undergoes runaway heating or cooling, generally grow on this time scale. The second time scale is the inflow time, i.e. the time it would take for turbulent stresses to cause a fluid element to drift inward over a distance comparable to its current radius,

$$
t_{\text {inflow }} \sim \frac{R}{v} \sim \frac{\Sigma \Omega R^{2}}{2 H w_{r \phi}} \sim \frac{\Omega}{\alpha \Omega_{\mathrm{K}}^{2}}\left(\frac{R}{H}\right)^{2} .
$$

"Viscous" or inflow instabilities tend to grow on this time scale, where I have enclosed the term "viscous" in quotation marks here (only) to emphasize that it is turbulent stresses, not microscopic viscosity, that play a role here. In geometrically thin accretion disks, where dynamical equilibrium on the inflow time requires the angular velocity and specific angular momentum of fluid orbits to be a function of radius and not of time, one can write down a time-dependent diffusion equation for the evolution of the surface mass density in the flow (Lynden-Bell \& Pringle 1974; Lightman \& Eardley 1974),

$$
\frac{\partial \Sigma}{\partial t}=\frac{1}{R} \frac{\partial}{\partial R}\left[\frac{1}{\ell^{\prime}} \frac{\partial}{\partial R}\left(R^{2} W_{R \phi}\right)\right],
$$

where $\ell^{\prime}=(d / d R)\left(R^{2} \Omega\right)$ is the radial derivative of specific angular momentum. In this case, instabilities happen if $W_{R \phi}$ is inversely related to the surface mass density, as this equation then corresponds to a diffusion equation with a negative diffusion coefficient. Perturbations in surface mass density would then tend to grow, rather than be smoothed out, by this anti-diffusion. 
Following theoretical work on dwarf nova outbursts in cataclysmic variables (e.g. Smak 1984), it has proved convenient to depict local thermal equilibrium $\left(Q^{+}=\right.$ $\left.Q^{-}+Q_{\text {adv }}\right)$ solutions at one particular radius in a diagram of accretion rate vs. local surface mass density. Figure 2 (Chen et al. 1995) depicts the topology of the space of such solutions at a radius of $10 G M / c^{2}$ around a ten solar mass Schwarzschild black hole. Each curve corresponds to a different chosen value of $\alpha$, as labelled. The locations of these curves in this graph can shift considerably, depending on the particular physics being included in the models, but the topological structure is robust. Naively, we expect equilibrium curves with negative slopes in this diagram to be viscously unstable, as the vertically integrated stress $W_{R \phi}$ is proportional to the dissipation rate per unit area $Q^{+}$ which in turn is proportional to the accretion rate $\dot{M}$, because, after all, it is the inflow of matter which is the source of accretion power. The negative sloped curve portions in the right of this diagram near Eddington accretion rates are radiation dominated, geometrically thin disks, and are viscously unstable by this criterion (Lightman \& Eardley 1974). They are also thermally unstable (Shibazaki \& Hōshi 1975; Shakura \& Sunvaev 1976), as can be seen because $Q^{+}$exceeds (is less than) $Q^{-}+Q_{\text {adv }}$ above (below) these curve portions. Hence a perturbation upward (downward) from this curve will cause runaway heating (cooling), moving it away from the equilibrium curve. Similarly, the middle bold line in the lower portion of this diagram, which corresponds to a hot, optically thin flow where turbulent dissipation is balanced by radiative cooling is viscously stable but thermally unstable. This solution was first discovered by Shapiro, Lightman \& Eardlev (1976).

This leaves three regions of the diagram which appear to correspond to thermally and viscously stable solutions. The lower right set of curves correspond to the gas pressure dominated regime of the original geometrically thin, optically thick accretion disks of Shakura \& Sunvaev (1973). At higher accretion rates radiation pressure starts to dominate these geometrically thin solutions and the curves bend over to the unstable negative slopes. At still higher accretion rates, the inflow time becomes shorter than the cooling time, so that heat is advected inward. This advection is stabilizing (Abramowicz et al. 1988) and, because the scale height of the disk can become quite large, these flows have been dubbed "slim disks". (Note that for larger radii, the optically thick, geometrically thin disk curves would also bend toward unstable negative slopes and then back toward positive stable slopes as one lowers the accretion rate and passes through the transition where ionized hydrogen becomes neutral. This unstable branch is responsible for the transient outburst behavior observed in many black hole and neutron star X-ray binaries, as well as dwarf novae in accreting white dwarf systems. We will have little to say about this instability here, as it generally occurs in the outer, less luminous portion of the disk in accreting black hole systems, but it is crucial for explaining the phenomenology of black hole transients. See Lasota 2001 for a good review of the theory. Note, however, that these outbursts provide some of the only constraints on the levels of turbulent stress in accretion disks, with $\alpha \simeq 0.1-0.3$ in the outburst phase. This is significantly higher than that measured in local shearing box simulations with no net vertical magnetic flux, as illustrated in Figure 1 above. It may be that the character of the turbulence changes when one is so close to the regime of hydrogen ionization, or it may be that external magnetic flux is necessary to explain the observations. This is a significant unsolved problem, e.g. King, Pringle \& Livio 2007; Kotko \& Lasota 2012.)

In addition to these two optically thick accretion disk solutions, a third set of stable solutions exists which is optically thin and which only exists at low accretion rates, 


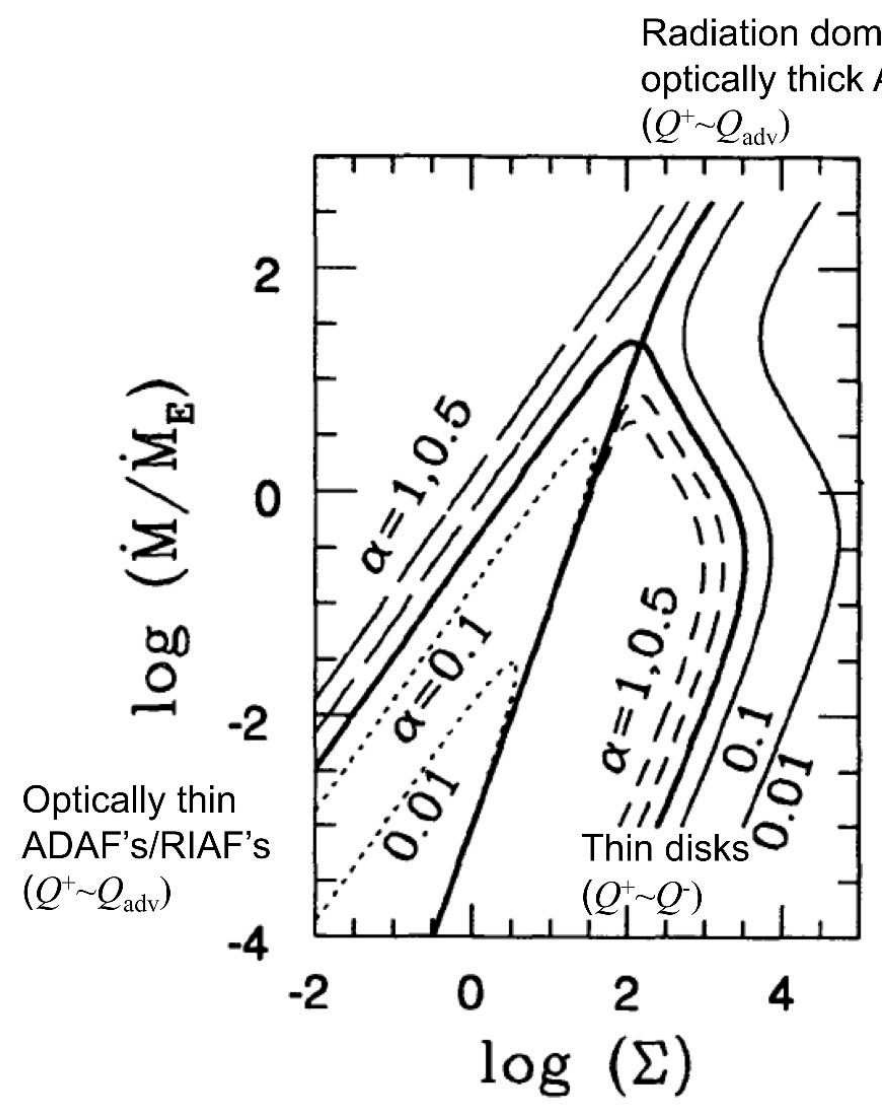

Fig. 2 Thermal equilibrium curves of particular accretion flow models around a ten solar mass black hole at a particular radius $10 G M / c^{2}$ on the accretion rate (scaled with $\dot{M}_{\mathrm{E}}$, the Eddington luminosity divided by $c^{2}$ ) vs. surface mass density $(\Sigma)$ plane, from Chen et al. (1995). Each curve is labelled by the value of the Shakura \& Sunyaev (1973) stress parameter $\alpha$ chosen in the model. The locations of these curves in this plane can change considerably depending on the physics being incorporated and how it is treated in these models - see Chen et al. (1995) for more details. The topology of the curves is, however, reasonably robust.

provided $\alpha$ is not too high. Here the cooling time significantly exceeds the infall time, so that advection again balances turbulent dissipation, and this apparently also stabilizes the flow (Ichimaru 1977; Naravan \& Yi 1994; Abramowicz et al. 1995; Chen et al. 1995; Naravan \& Yi 1995a 1995b). Such flows are known as Advection Dominated Accretion Flows (ADAF's, a term which can also be applied to the optically thick, radiation dominated slim disks) or radiatively inefficient accretion flows (RIAF's).

While the above arguments suggest that these three sets of solutions are thermally and viscously stable, attempts to rigorously demonstrate this involve subtle issues, particularly in the two advection dominated solutions, and the situation is in fact not entirely clear. Advection dominated solutions are fairly geometrically thick $(H \sim$ $R$ ), so the thermal and inflow time scales are comparable, and there is no longer a clean separation of thermal and viscous instabilities. Thermal pressure is also not dynamically negligible, so that thermal perturbations can alter the specific angular 
momentum distribution and the surface mass density in the disk, even without mass diffusion due to turbulent stresses. Attempts to tackle this problem have been made (e.g. Kato, Abramowicz \& Chen 1996; Wu 1996; Kato et al. 1997; Wu 1997; Yamasaki 1997), but they involve considerations of turbulent heat diffusion and turbulent bulk viscosity, for which there is currently very little understanding in the context of MRI turbulence. One hopes that simulations will shed light on these issues, and currently global MRI simulations in the RIAF regime appear to be consistent with thermal and viscous stability (see Chapter 2.4). As we discuss further below, simulations of the optically thick solutions, which are far more challenging, currently cast doubt on alpha prescription stability analyses, even in the supposedly unstable geometrically thin, optically thick radiation pressure dominated solution.

It should also be noted that, in addition to the thermal and viscous instabilities, other, shorter time scale instabilities exist that are driven by the thermodynamics of the alpha stress prescription, particularly the excitation of acoustic modes (e.g. Blumenthal. Yang \& Lin 1984; Kato. Honma \& Matsumoto 1988; Chen \& Taam 1993), and these might be relevant for explaining high frequency variability in black hole sources. Again, however, it is far from clear that the time-dependent thermodynamics of the alpha prescription accurately represents the time-dependent thermodynamics of MRI turbulence. On the other hand, dynamical excitation of nonaxisymmetric acoustic waves clearly occurs in MRI turbulence (Heinemann \& Papaloizou 2009a 2009b).

Each of the three solutions is expected to have a distinct relationship between overall radiative luminosity and accretion rate. Geometrically thin, radiatively efficient accretion disks are expected to have a luminosity which varies linearly with accretion rate, as for a given black hole spin, all the released binding energy is equivalent to an approximately fixed fraction of the rest mass energy of the accreted material. Advective models have reduced radiative efficiency. For the optically thin RIAF solutions, the luminosity is approximately proportional to the square of the accretion rate (Naravan \& Yi 1995b) and therefore the radiative efficiency drops as the accretion rate is reduced. Observational tests of this predicted relationship are discussed in Chapters 3.2 and 5.7. In the high luminosity regime of slim disks, the radiative output is expected to approximately saturate to the Eddington limit as photons become trapped (Begelman \& Meier 1982), and this is expected to remain true even if outflows are driven (e.g. Poutanen et al. 2007), although much of this luminosity will be emitted anistropically toward the rotation axis (e.g. Ohsuga \& Mineshige 2011; Chapter 5.3).

The three basic flow paradigms have been used over the years to explain the observed variety of black hole accretion sources. Something like the radiation pressure dominated advective slim disks are probably relevant for luminous quasars and QSO's ("quasi-stellar objects"), narrow line Seyfert 1's, ultraluminous X-ray sources, SS433, and perhaps some of the intermediate/steep power law states of black hole X-ray binaries. Geometrically thin, optically thick, radiatively efficient accretion disks extending down close to the central black holes are probably most relevant for the high/soft state of black hole X-ray binaries and perhaps for some QSO's. Optically thin RIAF's are almost certainly relevant for low luminosity active galactic nuclei (AGN), jet-dominated nonthermal AGN such as M87, the Galactic Center source Sgr $A^{\star}$, the inner regions of some broad line Seyfert 1's, and the inner regions of the low/hard state of black hole $\mathrm{X}$-ray binaries. Many of the other chapters in this book address how well these models work in explaining the observed properties of these sources. 
2.2 Tying local models into global models: the overall geometry of the flow

Figure 2 shows that, provided alpha is not too high, the RIAF solutions at any particular radius always terminate above a critical accretion rate where radiative cooling becomes comparable to turbulent dissipation (Abramowicz et al. 1995; Narayan \& Yi 1995b). This critical accretion rate generally decreases with radius at large radii, so that provided the accretion rate is not too low, the accretion flow will generally be in the radiatively efficient, geometrically thin disk state at large radii. However, at smaller radii, provided the accretion rate is not too high $\left(\dot{M} \lesssim 10 \alpha^{2} \dot{M}_{\mathrm{E}}\right.$, where $\dot{M}_{\mathrm{E}}$ is the Eddington luminosity divided by $c^{2}$; Naravan \& Yi $1995 \mathrm{~b}$ ), the accretion flow can exist in one of two distinct thermal equilibria: the optically thin RIAF solution, and the radiatively efficient geometrically thin disk solution. Narayan \& Yi (1995b) argue that evaporation from the surface of the thin disk will tend to drive the accretion flow into the RIAF regime whenever it is possible. One would then be left with a geometry which consists of an outer geometrically thin disk extending down toward a transition radius inside of which the flow adopts the RIAF solution. As the external accretion rate increases, the transition radius moves inward. This then provides an explanation for transitions between hard and soft states in black hole X-ray binaries (Esin. McClintock \& Naravan 1997, Figure 3). As discussed in Chapter 2.5, it is now well-established that the external accretion rate is not the only parameter that controls state transitions: hard to soft transitions generally occur at higher accretion rates than soft to hard transitions. Nevertheless, this geometry of an outer thin disk and an inner RIAF has become a popular model for hard states of black hole X-ray binaries, and of certain classes of active galactic nuclei.

RIAF's are not the only way to produce hard X-rays, however. As illustrated in the top most panel of Figure 3, it is possible that a corona containing hot or energetic nonthermal electrons exists above and below the geometrically thin disk. This could be locally generated by, for example, flares associated with buoyant magnetic field lines in a manner analogous to the production of the solar corona Galeev, Rosner \& Vaiana 1979; Haardt \& Maraschi 1991). Alpha disk models in which some fraction of the locally generated accretion power is dissipated in an external corona have been developed by Svensson \& Zdziarski (1994). Just as in the sun, the actual geometry of the corona could be quite complicated, with multiple coronal patches.

Starting with the work of Mever, Liu, \& Mever-Hofmeisten (2000a 2000b) and Różańska \& Czernv (2000), many attempts have also been made to build alpha-based models of outer thin disks, inner RIAF's, and coronae that themselves are treated as accreting RIAF's but which can sandwich portions of the thin disk. Each of the different flow regions exchange energy and mass through thermal conduction, evaporation and condensation, and irradiation. As shown in Figure 4, one can even form inner condensed pieces of radiatively efficient thin disks embedded inside the RIAF/corona flow in these models (e.g. Mever, Liu, \& Mever-Hofmeister 2007; Liu, Done \& Taam 2011). While such flow geometries may well occur in nature, using the same alpha prescription everywhere, especially at high latitudes off the midplane, might be problematic, as discussed briefly in section 3.2 below. One hopes that thermodynamically consistent global simulations of MRI turbulence may shed light on how transitions between thin disks, coronae, and RIAF's actually occur. 


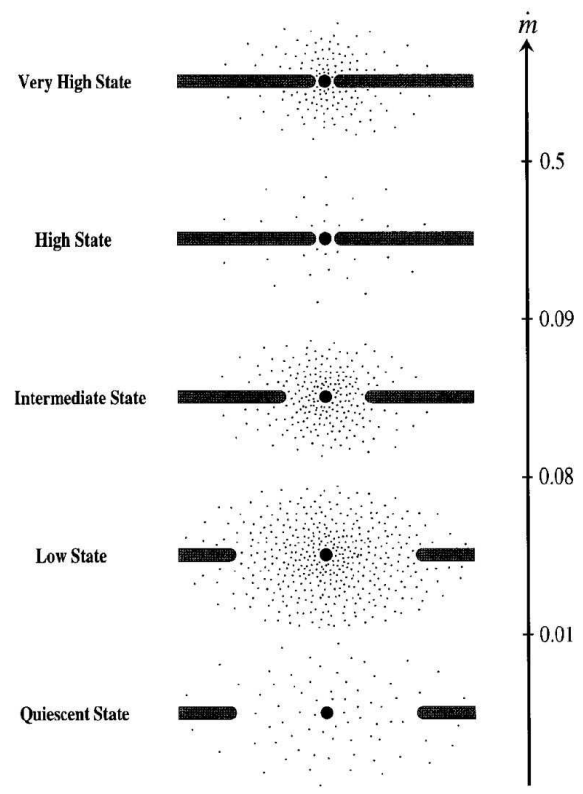

Fig. 3 Suggested flow geometries involving outer geometrically thin/optically thick disks and inner optically thin RIAF's for various observed states in black hole X-ray binaries, from Esin. McClintock \& Naravan (1997). Here $\dot{m}$ is the accretion rate scaled by ten times the Eddington luminosity divided by $c^{2}$.

\subsection{Other variants}

In addition to the three basic local models of optically thin RIAF's, radiatively efficient geometrically thin disks, and radiation pressure dominated advective slim disks, numerous other models have been proposed over the years. Some of these are variations on the three basic models, while others involve more significant departures from the physics included in these models.

Because much of the dissipated accretion power is not radiated away, both the optically thin and optically thick advection dominated solutions involve fluid which is only weakly bound to the black hole, i.e. with internal energy comparable in magnitude to the orbital binding energy. Outflows are therefore very likely to occur in these regimes (Naravan \& Yi 1994; Blandford \& Begelman 1999), and one way of incorporating them (with little physics beyond the invocation of self-similarity) is to simply assume that the accretion rate varies as a power law with radius $\dot{M} \propto R^{p}$ (Blandford \& Begelman 1999). Assuming accretion velocities scale with the free-fall speed $\propto R^{-1 / 2}$, as self-similarity would require, such solutions have a radial density profile $\rho \propto R^{p-3 / 2}$, with $p=0$ corresponding to the standard ADAF. Such solutions have been dubbed Advection-Dominated Inflow-Outflow Solutions (ADIOS) by Blandford \& Begelman (1999). Outflows have been commonly observed in global simulations of these flow regimes, (Chapters 2.4 and 5.3), though exactly how much mass 


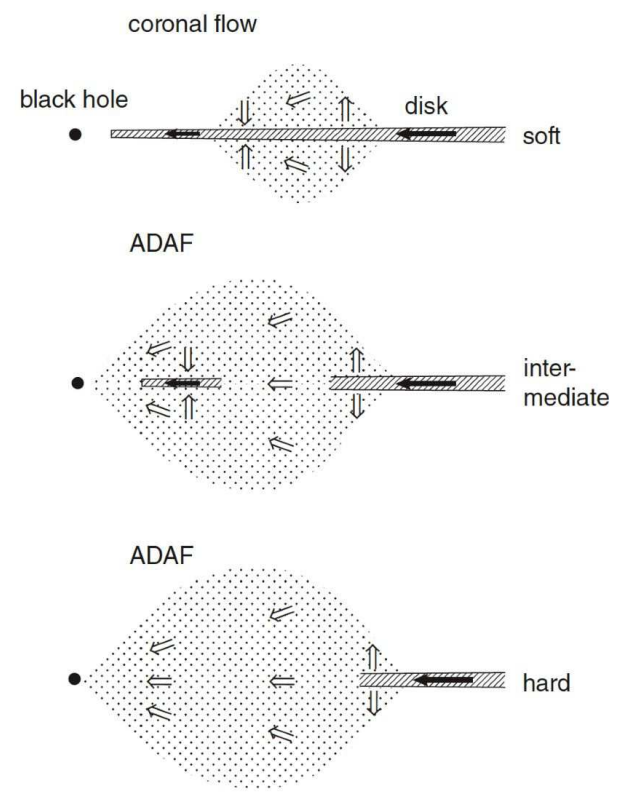

Fig. 4 Possible accretion flow geometries in evaporation/condensation models, from Meyer, Liu, \& Mever-Hofmeister (2007).

is lost compared to how much is accreted remains theoretically uncertain. Very recent simulations by Naravan et al. (2012) in the low luminosity RIAF regime find that outflows are not as powerful as previously thought. Time-dependent self-similar ADAF solutions by Ogilvie (1999), that differ from the stationary self-similar solution of Narayan \& Yi (1994), appear to be consistent with this result. At high luminosities, outflows can also be driven directly by radiation pressure, particularly with the high atomic opacities expected for gas around supermassive black holes in active galactic nuclei (Murrav et al. 1995; Proga. Stone \& Kallman 2000).

Another consequence of not dissipating accretion power in advection dominated flows is that the entropy of the plasma increases inward, implying that the flow will be, at least hydrodynamically, convectively unstable. It has been suggested that large scale convection in the flow can transport angular momentum inward, and stationary solutions in which only small accretion rates occur as material circulates again and again in convective eddies have been proposed which have radial density profiles $\rho \propto R^{-1 / 2}$ (Convection Dominated Accretion Flows or CDAF's; Naravan. Igumenshchev \& Abramowicz 2000; Quataert \& Gruzinov 2000). The physical consistency of such solutions is controversial (Balbus \& Hawley 2002; Naravan et al. 2002). The recent RIAF simulations by Naravan et al. (2012) also do not find obvious signs of convection.

Yet another variant on the optically thin RIAF model is the luminous hot accretion flow (LHAF) model of Yuan (2001). As we discussed above, unless alpha is large, RIAF's have a maximum accretion rate above which the solutions with advective cool- 
ing do not exist. This creates a problem for using them to explain hard state sources that are observed to exist at high luminosities. The LHAF model solves this problem by positing that advective cooling is replaced by advective heating. The heating here is due to compressional work, and is non-dissipative. LHAF's therefore essentially balance compressional work plus turbulent dissipation with radiative cooling. However, such models appear to be thermally unstable given their local equilibrium curves on the accretion rate vs. surface mass density plane, although the growth rates might be long enough to not significantly affect the flow (Yuan 2003).

Models involving strong and/or large-scale magnetic fields in the flow have also been proposed, for example flows in which magnetic pressure dominates thermal pressure (Pariev, Blackman \& Boldvrev 2003; Meier 2005; Machida, Nakamura, \& Matsumoto 2006; Begelman \& Pringle 2007), and flows in which angular momentum transport is dominated by large-scale MHD outflows (e.g. Ferreira \& Pelletier 1995). It is even possible for enough magnetic field to be advected inward in the accretion flow that it becomes strong enough to disrupt the flow, allowing accretion to only proceed through magnetic Rayleigh-Taylor interchange motions (so-called Magnetically Arrested Disks; Naravan. Igumenshchev \& Abramowicz 2003, see also Chapter 2.4). Such flows are beyond the scope of this particular review, which focuses on accretion driven by turbulence, although they may very well be important in nature. It is now time for us to turn to what is known about the local properties of MRI turbulence in accretion flows.

\section{Going beyond alpha: MRI turbulence}

Since the discovery of its relevance to the physics of accretion flows (Balbus \& Hawley 1991; Hawley \& Balbus 1991), tremendous theoretical effort has been expended to try and understand the properties of MRI turbulence in various regimes of relevance to astrophysics. Studying MRI turbulence has allowed us to pose questions that simply cannot be asked within the alpha prescription, and has led to considerable new physical insight into how the accretion process works, as well as sharpened the true physical uncertainties. It is, after all, only by building models based on real physics, rather than precriptions that sweep undertainties into a single parameter, that real scientific progress can be made. However, it has to be admitted that the ultimate goal of replacing alpha-based modeling of accretion powered sources with observationally falsifiable models based on the actual physics of the turbulence has not yet been achieved. In this section I will review some of the fundamental physics issues that have been understood, or at least revealed, by local simulations of MRI turbulence. Global MRI simulations of black hole accretion flows have also provided considerable insight, and are in fact closest to realizing the goal of providing observationally testable models of the accretion flow onto the Galactic Center black hole source Sgr A*. These global simulations are reviewed in Chapter 2.4.

\subsection{Shearing box simulations of MRI turbulence}

It is in the very nature of fully nonlinear, strong turbulence that energy released or injected from large spatial scales passes quickly down to microscopic dissipation scales through a turbulent cascade. In our case gravitational binding energy is released through the MRI which grows by tapping directly into the free energy associated with 
the differential rotation inherent in the accretion flow. The microscopic dissipation scales are associated with the true viscosity and resistivity of the plasma. The actual physical dissipation scales relevant to black hole accretion flows are extremely small compared to the energy release scales of the MRI (presumably of order the disk thickness), but numericists have nevertheless hoped that by putting in enough grid zones into their simulations, that some convergence can be achieved in describing the properties of MRI turbulence. That hope is best achieved in local shearing box simulations of the turbulence, where all the computing power is devoted to resolving scales within the turbulent cascade, and not on the larger scale dynamics associated with the overall flow geometry (as important as these larger scales are to ultimately understanding observed sources).

The geometry and properties of the shearing box are very nicely described by Hawley, Gammie \& Balbus (1995). Essentially a small, perfectly rectangular Cartesian box is placed in the rotating shear flow, and corotates with the background flow at the center of the box. The curvature in the background flow streamlines are entirely neglected, but the effects of rotation are nevertheless included through Coriolis forces as well as centrifugal forces that are combined with the gravitational force through an effective potential. Boundary conditions are such that the flow is assumed to be perfectly periodic in the azimuthal direction, but shearing periodic in the radial direction: one imagines many identical shearing boxes sliding past each other according to the background differential rotation. If the box is placed in the midplane of the flow, one sometimes neglects the vertical gravity and adopts periodic boundary conditions in the vertical direction (unstratified shearing boxes), but one can also include vertical gravity (stratified shearing boxes) and adopt outflow boundary conditions, or, for computational convenience, retain vertical periodic boundary conditions (a stack of accretion disk pancakes!).

The symmetries of the standard shearing box mean that there is no net accretion of mass through the box, and therefore in fact no release of gravitational binding energy. All the energy associated with the turbulence in fact arises from the net work done by the turbulent stresses on the shearing walls of the box.

Any initial net vertical magnetic field must be conserved in a shearing box simulation, and this is also true of net azimuthal magnetic field if the shearing box is unstratified (such field can leak out of the vertical boundaries of stratified boxes if outflow boundary conditions are employed). Shearing box simulations can therefore have the net magnetic flux through the box as a fixed external parameter. Unstratified simulations with no external flux, and no explicit treatment of viscosity and resistivity which would resolve dissipation on scales larger than the grid scale, actually produce MRI turbulent stresses that monotonically decrease with increasing numerical resolution (Pessah. Chan \& Psaltis 2007; Fromang \& Papaloizou 2007)! However, this is a singular situation, as including explicit viscosity and resistivity in the MHD equations (albeit at far larger values than are relevant for black hole accretion flows) or a net magnetic flux does lead to converged levels of stress as numerical resolution is increased. This stress increases with the amount of external magnetic flux (Hawlev. Gammie \& Balbus 1995; Pessah. Chan \& Psaltis 2007) and also increases with the dimensionless ratio of kinematic viscosity to Ohmic resistivity, known as the magnetic Prandtl number (Lesur \& Longaretti 2007; Fromang et al. 2007; Simon \& Hawlev 2009). The result that stress increases with net magnetic flux has also been confirmed in localized regions of global simulations (Sorathia, Reynolds \& Armitage 2010). 


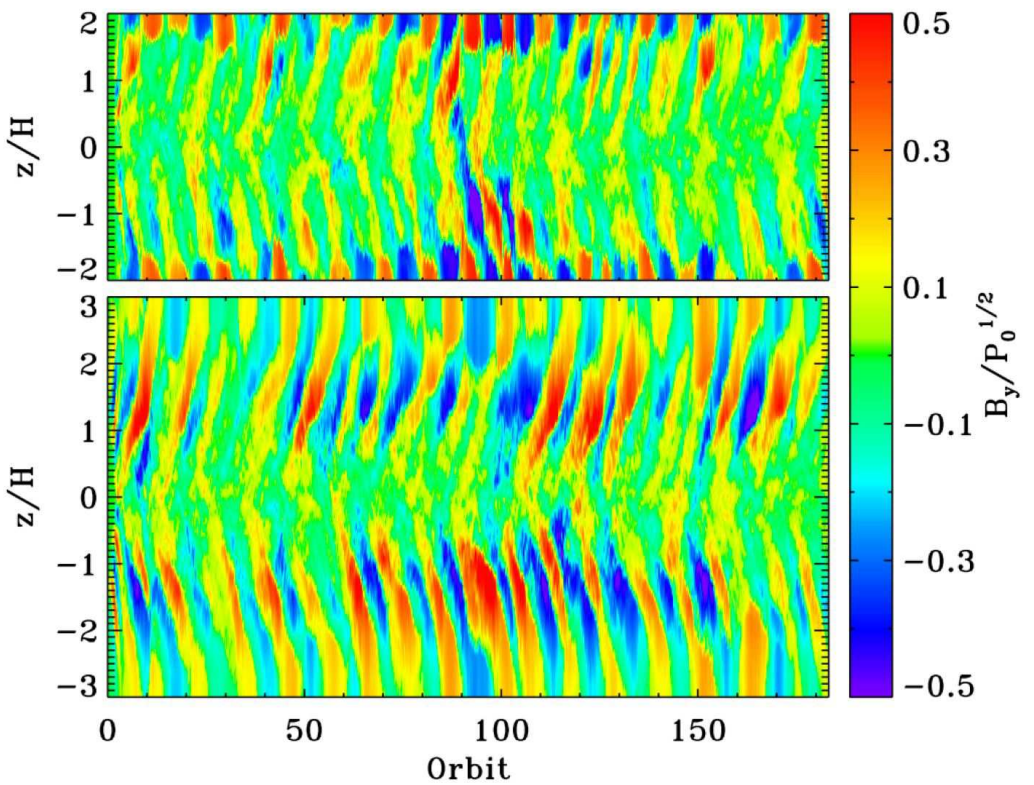

Fig. 5 Horizontally-averaged azimuthal component of the magnetic field as a function of height $z$ and time in two vertically stratified shearing box simulations whose only difference is the height of the box, from Davis, Stone \& Pessah (2010). There exists a dynamo in such vertically stratified simulations that causes quasiperiodic azimuthal field reversals.

Adding in vertical gravity in stratified simulations enables convergence of the turbulent stresses even without net magnetic flux and explicit viscosity and resistivity (Shi. Krolik \& Hirose 2010; Davis, Stone \& Pessah 2010). A major difference between unstratified and stratified shearing box simulations is that gravity allows for magnetic buoyancy, and this is clearly playing a role as alternating signs of azimuthal field continually develop in a dynamo within the MRI turbulence and rise outward (Brandenburg et al. 1995). For weak magnetic fields, this produces a quasiperiodic pattern of field reversals, as shown in Figure 5. (When time-reversed, the pattern here resembles the butterfly diagram of latitudunal distributions of sunspots over the course of the solar cycle. By analogy, this behavior is occasionally referred to as the MRI butterfly diagram.) Moreover, stratified shearing box simulations with vertical outflow boundary conditions and net vertical magnetic flux can actually locally produce magnetocentrifugally driven outflows (Suzuki \& Inutsuka 2009; Fromang et al. 2012; Lesur, Ferreira \& Ogilvie 2012; Bai \& Stone 2012). Increasing the net vertical magnetic flux in such simulations can also destroy the periodicity of the MRI butterfly dynamo, and ultimately suppress it (Bai \& Stone 2012).

The whole premise of the alpha prescription is that the stresses are inherently local: (vertically-averaged) stress just depends on (vertically-averaged) thermal pressure. This appears to be mostly confirmed by shearing box simulations which have wide radial extents, in that spatial correlations between stresses at different locations de- 
crease rapidly on radial scales larger than the disk scale height. However, there remain $\sim 20$ percent correlations in the Maxwell stress on larger radial scales, indicating that the turbulence may not be entirely local (Simon, Beckwith \& Armitage 2012). The butterfly dynamo cycles have also been observed in global simulations and have significant radial coherence on scales much larger than a disk scale height (O'Neill et al. 2011).

\subsection{Aspects of the vertical structure revealed by MRI simulations}

Provided any external magnetic flux is not too high, stratified shearing boxes generally result in a structure that is dominated by thermal pressure in the midplane and magnetic pressure and tension forces in the outer layers (Stone et al. 1996; Miller \& Stone 2000; Hirose. Krolik \& Stone 2006). MRI turbulence is generally confined to the weakly magnetized regions near the midplane, while Parker instability dynamics dominates the outer regions (Blaes. Hirose \& Krolik 2007). This basic structure of weakly magnetized midplane regions and more strongly magnetized high altitude regions is also generally observed in global simulations (e.g. Hawley \& Balbus 2002; Penna et al. 2010; Sorathia, Reynolds \& Armitage 2010), although here the strongly magnetized regions at high altitude can involve significant radial flows and circulation, which cannot happen in a shearing box. The fact that the magnetic pressure profile is broader than the thermal pressure profile, and that Maxwell stresses generally dominate Reynolds stresses in the turbulence, implies that an average of the ratio of stress to thermal pressure (alpha!) generally increases outwards: alpha should never be treated as a local quantity, but instead it is, at best, a representation of the ratio of vertically-averaged stress to pressure. Disk atmospheres are generally supported by magnetic fields, not thermal pressure, so that if atmosphere models of thermal and reflection spectra rely critically on vertical hydrostatic equilibrium between gravity and thermal pressure gradients, they may not be accurate. In addition, the fact that MRI turbulence is generally confined to the weakly magnetized midplane regions suggests that models of accreting coronal flows discussed in section 2.2 above are probably not well described by a simple alpha prescription.

The magnetically-dominated outer layers are very suggestive of the locally generated magnetized corona discussed above in section 2.2. Indeed, very tall stratified shearing box simulations by Miller \& Stone (2000) found that approximately a quarter of the magnetic energy generated in the turbulent midplane regions was carried out into the corona. These simulations assumed an isothermal equation of state, however, and vertically stratified simulations that capture turbulent dissipation as heat and incorporate diffusive radiation transfer have generally found that the fraction of accretion power that is dissipated in the magnetized corona outside the photosphere is very small (Hirose, Krolik \& Stone 2006; Krolik, Hirose \& Blaes 2007; Hirose, Krolik \& Blaes 2009). On the other hand, the magnetic buoyancy exhibited in the butterfly diagram illustrated in Figure 5 can be very energetically important in transporting significant amounts of thermal energy outward in the form of trapped photons in the radiation pressure dominated regime (Blaes et al. 2011). It should also be emphasized that the existing radiation MHD simulations have not included a net vertical magnetic flux, which might in principle enhance the coronal energetics. 
3.3 Physics issues in the RIAF regime

Low luminosity RIAF models continue to be plagued by significant uncertainties in the microphysics of the plasma, whether they are globally simulated with MRI turbulence or modeled with an alpha prescription. Because the accreting plasma retains a significant fraction of its binding energy as internal energy, temperatures must approach virial temperatures: $k T \sim G M m_{\mathrm{p}} / R=\left(R_{\mathrm{g}} / R\right) m_{\mathrm{p}} c^{2}$, where $m_{\mathrm{p}}$ is the proton mass. This corresponds to $\sim 10^{12} \mathrm{~K}$ at ten gravitational radii. Optically thin cooling by electrons at such temperatures is very fast, unless the accretion rate and density is extremely low, so in order to not radiate away all the heat on an inflow time, the electron temperature $T_{\mathrm{e}}$ must be significantly less than the ion temperature $T_{\mathrm{i}}$. This in turn implies that the electrons should not receive the vast majority of the turbulent dissipation of accretion power (otherwise they would radiate it away), and the ions must be at least partially thermally decoupled from the electrons on the inflow time. Coulomb collisions alone will be insufficient to thermally couple ions and electrons provided the accretion rate is not too high (Rees et al. 1982; Narayan \& Yi 1995b). Plasma instabilities may in principle exist that couple the species more rapidly, but so far this has not been demonstrated (Begelman \& Chiueh 1988; Park et al. 2010).

Even the MRI behaves differently in the collisionless regime (Quataert, Dorland \& Hammett 2002; Sharma. Hammett, \& Quataert 2003; Balbus 2004; Islam \& Balbus 2005), especially in giving rise to anisotropic pressure tensors that themselves can give rise to significant angular momentum transport (Sharma et al. 2006), and this is not captured in simulations that assume MHD. How the turbulent dissipation is ultimately channeled into heating of the ions and electrons (or energization - the ion and electron distribution functions need not be thermal) is another significant uncertainty. Local simulations find that direct heating by the anisotropic pressure tensor can account for 50 percent of the heating by the turbulence, and that the ratio of electron to ion heating is $\sim 0.3\left(T_{\mathrm{e}} / T_{\mathrm{i}}\right)^{1 / 2}$ (Sharma et al. 2007). It is also just now becoming possible to do fully kinetic simulations of collisionless MRI turbulence, at least locally (Riquelme et al. 2012), so that there is hope for further resolving some of these issues in the not so distant future.

All these effects remain to be included in global simulations of the RIAF regime, discussed in Chapter 2.4. Currently these generally assume regular MHD, and adopt prescriptions for treating the electron and ion distribution functions, such as assuming thermal distributions with a constant ion to electron temperature ratio.

3.4 Attempts to simulate the radiation pressure thermal instability

For some years now, it has been possible to do vertically stratified shearing box simulations that explore the thermodynamics of MRI turbulence. These simulations capture grid scale losses of magnetic and kinetic energy, and incorporate radiation transport and cooling through flux limited diffusion. It has also been possible to do global simulations using flux limited diffusion under axisymmetry - see Chapter 5.3. Recently, even more accurate radiation transport algorithms have been successfully developed (e.g. Davis, Stone \& Jiang 2012; Jiang, Stone \& Davis 2012; Sadowski et al. 2012; Takahashi et al. 2012).

Vertically stratified shearing box simulations with optically thick cooling and which incorporate the dynamics of radiation pressure enable the exploration of the thermal 
instability predicted by alpha disk modeling on the negative slope branch of the thermal equilibrium curves on the right hand side of Figure 2. The origin of this thermal instability is very easy to understand. Assuming that radiation diffusion dominates the vertical heat transport (which is generally found in simulations), the local cooling rate per unit area is of order the radiation energy density at the midplane $a T^{4}$ times the speed of light over the vertical optical depth. Because the opacity is dominated by electron scattering in these high temperature regimes, this then implies that $Q^{-} \propto T^{4} / \Sigma$. On the other hand, the heating rate per unit area is the vertically integrated stress times the rate of strain, $Q^{+} \sim H w_{R \phi} R|d \Omega / d R|$. Because the disk is supported vertically by radiation pressure, and the vertical gravity increases linearly with height above the midplane, the disk half thickness is simply proportional to the surface radiation flux, i.e. $H \propto Q^{-} \propto T^{4} / \Sigma$. Hence a standard alpha prescription, in which $w_{R \phi}=\alpha P=\alpha a T^{4} / 3$, will mean that the heating rate $Q^{+} \propto T^{8} / \Sigma$. Because the inflow time is much longer than the thermal time for geometrically thin disks, the surface mass density $\Sigma$ cannot vary significantly on the thermal time scale, and the heating rate therefore depends much more sensitively on temperature than the cooling rate. Hence a perturbative increase (decrease) in temperature would lead to runaway heating (cooling).

As shown in Figure 1. simulations are consistent with the standard alpha prescription that the time and space averaged stress scales with total thermal pressure, which is mostly radiation pressure in this regime. Nevertheless, at least some simulations have been able to establish thermal equilibria between heating and cooling in the radiation pressure dominated regime that last for many thermal times (Turner 2004; Hirose. Krolik \& Blaes 2009). On the other hand, recent simulations by Jiang. Stone \& Davis (2013b) find that such equilibria, even if established, always eventually suffer runaway heating or cooling. It is not clear what is producing the differences between the different simulations, which are run using different codes.

Note that, in contrast to the hydrogen ionization driven thermal/viscous instability that is responsible for dwarf nova and outbursts in X-ray binaries, there is no similar observational evidence for the putative radiation pressure driven thermal instability predicted by alpha disk theory. It could be that the intrinsically stochastic nature of the turbulent dissipation, which is not captured in the alpha prescription, acts as a stabilizing influence (Janiuk \& Misra 2012). But in addition, it is clear that the alpha prescription breaks down on short time scales. As shown in Figure 6. fluctuations in thermal energy lag fluctuations in turbulent energy by approximately a thermal time. This is easy to understand physically: it is the dissipation of turbulence that heats the plasma and produces thermal pressure, and thermal energy will therefore only respond to fluctuations in turbulent energy on time scales of order the heating time $\sim(\alpha \Omega)^{-1}$. There appears to be no direct feedback from pressure to stress on the thermal time scale, and the alpha prescription is therefore only established on longer time scales, although exactly why the prescription is established remains mysterious. More thought needs to be applied to understanding the relationship between stress and pressure, and to understanding what is going on to explain the different results seen in different simulation codes. Recently, Lin et al. (2012) have taken a useful first step in developing analytic, time-dependent ordinary differential equations that successfully reproduce a number of the observed features in these simulations.

Note that while the thermal instability has been studied using local simulations of MRI turbulence, the inflow or viscous instability has not as the boxes that have been 


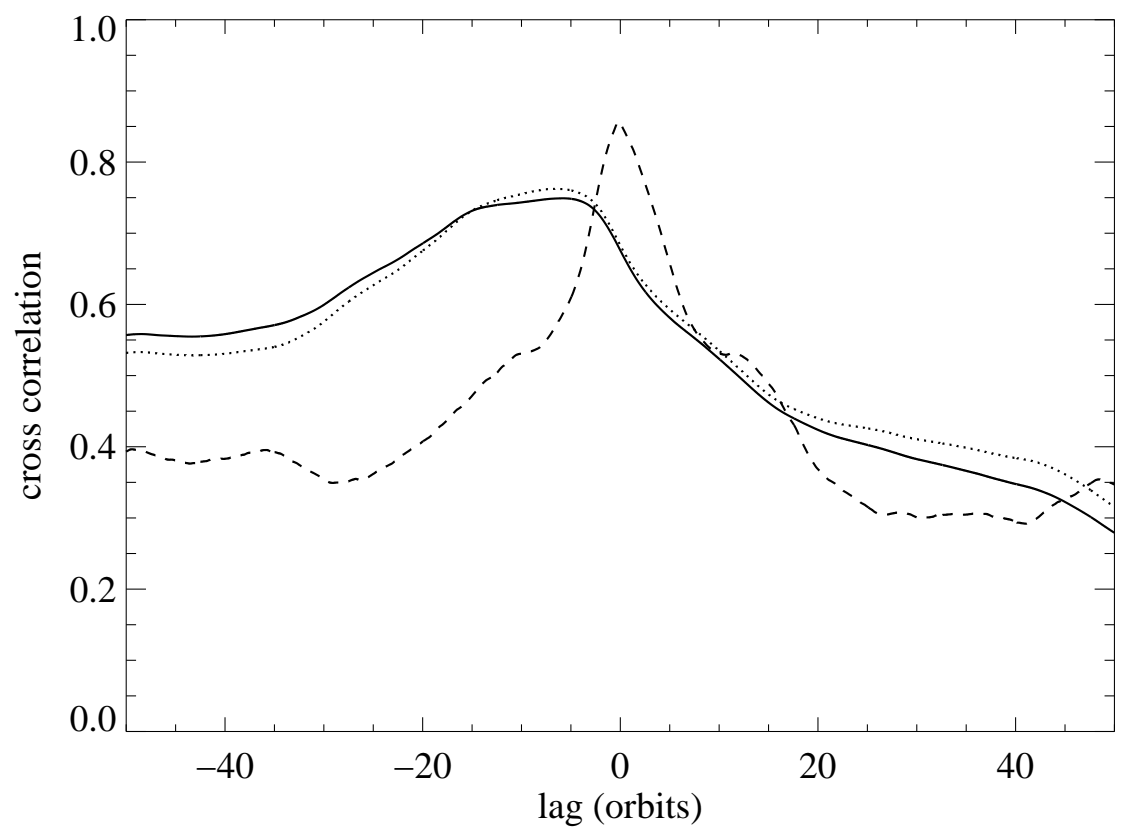

Fig. 6 Cross correlation coefficient for various forms of volume integrated energy as a function of time difference with respect to variations in volume integrated magnetic energy, for a radiation dominated stratified shearing box simulation. Negative values on the horizontal axis mean that the energy lags behind magnetic energy. The dashed line shows the turbulent kinetic energy, which is highly correlated with magnetic energy at zero lag. This is because both energies are aspects of the same MRI turbulence! The solid and dotted curves show radiation and gas internal energies, respectively. Both of these are very similar as they are both thermal energies, and both are again correlated with magnetic energy, but with a significant time lag of order 5-15 orbital periods. This is comparable to the thermal time in this simulation. (From Hirose, Krolik \& Blaes 2009.)

used are radially too narrow to allow for significant variations in surface mass density. Very wide radial boxes, or global simulations, will be necessary to explore this physics.

3.5 Other issues in the radiation dominated regime

Radiation dominated plasmas have a number of other interesting properties that are likely relevant to black hole accretion flows in the high luminosity regime. One in particular is that the sound speed in the fluid $\simeq\left[4 a T^{4} /(9 \rho)\right]^{1 / 2}$ is determined by radiation pressure, not gas pressure, and when the former exceeds the latter, it is possible to be in a regime where turbulent motions are subsonic and yet supersonic with respect to the sound speed from gas pressure alone. But photons generally diffuse through the plasma, and if they do so rapidly, then even fluid motions that are subsonic with respect to the radiation pressure sound speed, but supersonic with respect to the gas sound speed, can be highly compressible, because photon diffusion reduces the photon pressure response. Large density fluctuations can therefore be produced, and 
this has been observed in unstratified shearing box simulations of radiation dominated MRI turbulence (Turner et al. 2003; Jiang. Stone \& Davis 2013a).

Radiation damping of temperature fluctuations in radiation pressure dominated MRI turbulence can be a significant source of dissipation which, like the pressure anisotropies in collisionless MRI turbulence discussed above in section 3.3, can be resolved in numerical simulations. Such fluctuations can be compressible in nature as we just mentioned (Agol \& Krolik 1998), or due to nonlinear isobaric fluctuations associated with regions of high magnetic pressure (Blaes et al. 2011). Some tens of percent of the total dissipation has been observed to occur through radiation damping in shearing box simulations (Turner 2004; Blaes et al. 2011). This radiation damping can also increase the bulk viscosity and therefore the magnetic Prandtl number, and can increase the Maxwell stress in the turbulence (Jiang. Stone \& Davis 2013a).

The fact that MRI turbulence can be supersonic with respect to the sound speed in the gas alone in the radiation dominated regime implies that, in principle, turbulent speeds can exceed mean thermal speeds not only of the ions in the plasma, but also the electrons. If turbulent motions are limited by the radiation sound speed then this may start to happen at radiation to gas pressure ratios in excess of the proton to electron mass ratio, and may happen at even lower ratios in the photosphere regions which tend to be dominated by magnetic pressure, not thermal pressure. Differences in bulk turbulent velocities on the scale of a photon mean free path that exceed in magnitude characteristic electron thermal speeds will mean that bulk Comptonization by the turbulence itself will dominate thermal Comptonization, and this may provide an alternative means of producing a Comptonized high energy spectrum in radiation dominated luminous states of black hole sources (Socrates, Davis \& Blaes 2004; Socrates 2010).

Advection of heat is a key ingredient to the radiation pressure dominated slim disk solutions discussed in section 2.1 above. However, instabilities might produce inhomogeneities in the flow that allow photons to escape more readily through underdense channels, rather than be advected inward. The most well-explored of such instabilities are magnetically-mediated "photon bubble" instabilities (Arons 1992; Gammie 1998). On short length scales where photons diffuse rapidly, such instabilities amount to radiatively amplified magnetosonic modes (Blaes \& Socrates 2003) that develop into highly inhomogeneous trains of shocks (Begelman 2001; Turner et al. 2005, see Figure 7). In principle such inhomogeneities could allow locally super-Eddington fluxes to escape from the disk atmosphere without driving an outflow (Begelman 2002). Simulating photon bubbles in the presence of MRI turbulence has proved computationally challenging in the radiation pressure dominated regime due partly to the small length scales (of order the gas pressure scale height) that must be resolved, and partly by the fact that Parker instabilities in the magnetically dominated surface layers also produce significant inhomogeneity. Models of slim disks with porous outer layers and winds have recently been developed by Dotan \& Shaviv (2011). It has also been suggested that accretion flows in the radiation dominated regime might be highly inhomogeneous structures that are not well-described by any of the standard accretion flow models discussed in section 2.1 (Dexter \& Agol 2011). 

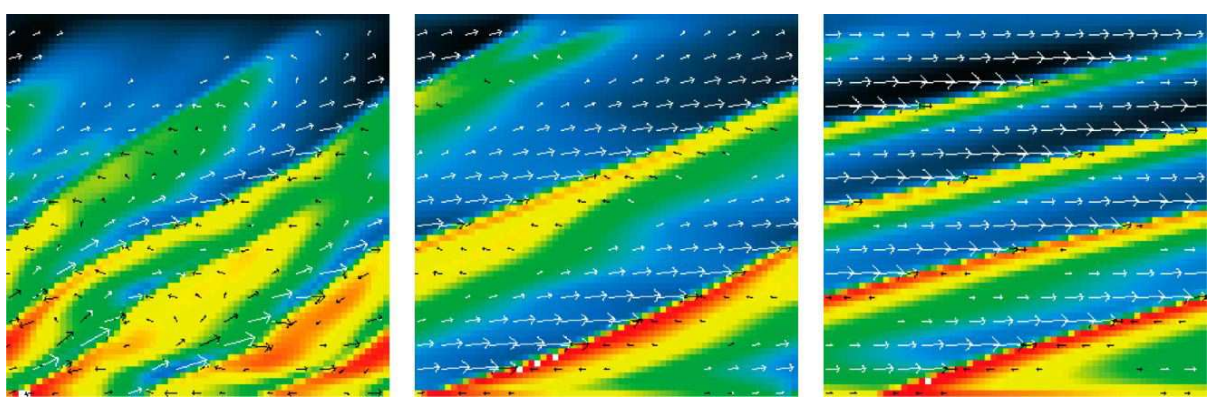

Fig. 7 2D simulations of shock trains produced by the photon bubble instability in radiation pressure supported media with initially uniform magnetic fields of increasing strength from left to right. Arrows show fluid velocity and colors show density on a logarithmic scale, with warm colors being high and cold colors being low. The weight of the dense shocked fluid causes the weaker magnetic fields in the left hand figure to buckle. (From Turner et al. 2005.)

\section{Summary}

The primary reason why the alpha prescription continues to be a mainstay of black hole accretion theory is that it enables models to be built that couple the dynamics of the flow (outward angular momentum transport through the plasma by a stress described phenomenologically by alpha) to the thermodynamics (dissipation of accretion power described phenomenologically by the same alpha stress prescription times the shear rate). Once this is all combined with radiative cooling processes, one has models that can be used to generate spectra, time variability, etc. that can be compared to observations. While decades of theoretical work within this research paradigm have yielded valuable insights (e.g. scalings of luminosity and temperature with accretion rate, the importance of advection of heat), one can only carry this program so far without addressing the fundamental physics that the alpha prescription hides.

(Fewer) decades of research have now been spent on understanding the properties of MRI turbulence, but until recently, this has focused mostly on the dynamics of the turbulence, not the thermodynamics, and it is the latter which is ultimately required to connect to observations, which are, after all, detecting the photons emitted by the source. We still have a lot of unanswered fundamental questions. For example, what is the true nature of the thin disk/RIAF transition radius, upon which hangs so much phenomenology of black hole X-ray binaries (hard/soft state transitions, band-limited noise, low frequency quasi-periodic oscillations, ...)? The thermal/viscous instabilities have guided theoretical effort to exclude uphysical equilibria, but are these instabilities always real, and how do they manifest? (Only those driven by ionization/recombination are observationally known to exist, e.g., in dwarf novae.) What determines how accretion power is partitioned into various forms? These are all questions of thermodynamics, not just dynamics.

As discussed in Chapter 2.4, global MRI simulations of low luminosity RIAF's have been most successful in connecting to observations, largely because, at least in some regimes, the radiative cooling does not dramatically affect the dynamics of the flow, and therefore can be calculated after the fact by post-processing the simulation. Moreover, because the flow is optically thin at most photon frequencies, the simulation hardware has actually developed to the point where radiative cooling can be fully incorporated in the actual dynamical simulation itself (Dibi et al. 2012). However, as discussed in 
section 3.3 above, even here there remain issues in the microphysics that still need to be understood theoretically.

Optically thick radiation transport is computationally more expensive, and also more important for the dynamics in the high luminosity black hole accretion regimes where radiation pressure plays a critical role. As discussed in section 3.4 above, local shearing box simulations have shed some light on how MRI turbulence works in this regime, and in what ways the alpha prescription does, and does not, describe the physics. Unfortunately, this still leaves major unanswered questions as to the global structure of the flow. Two dimensional (axisymmetric) global simulations have also been done which have confirmed the existence of discrete flow states (Ohsuga et al. 2009; Ohsuga \& Mineshige 2011, see also Chapters 2.4 and 5.3), but these cannot be run over long time scales as MRI turbulence cannot be sustained in axisymmetry. With the ongoing increase of computer power, combined with the development of new radiation transport algorithms, it should be possible to do global $3 \mathrm{D}$ simulations of accretion flows in optically thick regimes too. This will better enable us to understand the origins of state transitions in black hole X-ray binaries, transitions between radiatively efficient thin disks and RIAF's, and other fundamental problems in black hole accretion flows. Achieving this goal should complete the shift to a more physics-based research paradigm.

Acknowledgements The author is grateful to ISSI and to the organizers of this workshop for enabling such a productive set of scientific interactions, and thanks a number of the participants, particularly Chris Fragile, Tom Maccarone, Shin Mineshige, Ken Ohsuga, Juri Poutanen, and Chris Reynolds, for enlightening him on various issues of relevance to this paper. The author is also grateful to the referees for suggestions that significantly improved the manuscript. The author's research is supported by the US National Science Foundation under grant AST0707624 .

\section{References}

M. A. Abramowicz, B. Czerny, J. P. Lasota, E. Szuszkiewicz, ApJ, 332, 646-658 (1988)

M. A. Abramowicz, X. Chen, S. Kato, J. P. Lasota, O. Regev, ApJ, 438, L37-L39 (1995)

E. Agol, J. Krolik, ApJ, 507, 304-315 (1998)

J. Arons, ApJ, 388, 561-578 (1992)

X.-N. Bai, J. M. Stone, ApJ, submitted (2012), arXiv:1210.6661

S. A. Balbus, ApJ, 616, 857-864 (2004)

S. A. Balbus, J. F. Hawley, ApJ, 376, 214-222 (1991)

S. A. Balbus, J. F. Hawley, ApJ, 400, 610-621 (1992)

S. A. Balbus, J. F. Hawley, Rev. Mod. Phys., 70, 1-53 (1998)

S. A. Balbus, J. F. Hawley, ApJ, 573, 749-753 (2002)

S. A. Balbus, J. C. B. Papaloizou, ApJ, 521, 650-658 (1999)

M. C. Begelman, ApJ, 551, 897-906 (2001)

M. C. Begelman, ApJ, 568, L97-L100 (2002)

M. C. Begelman, T. Chiueh, ApJ, 332, 872-890 (1988)

M. C. Begelman, D. L. Meier, ApJ, 253, 873-896 (1982)

M. C. Begelman, J. E. Pringle, MNRAS, 375, 1070-1076 (2007)

O. Blaes, A. Socrates, ApJ, 596, 509-537 (2003)

O. Blaes, S. Hirose, J. H. Krolik, ApJ, 664, 1057-1071 (2007)

O. Blaes, J. H. Krolik, S. Hirose, N. Shabaltas, ApJ, 733, 110, 24 pp. (2011)

R. D. Blandford, M. C. Begelman, MNRAS, 303, L1-L5 (1999)

R. D. Blandford, D. G. Payne, MNRAS, 199, 883-903 (1982)

G. R. Blumenthal, L. T. Yang, D. N. C. Lin, ApJ, 287, 774-784 (1984)

A. Brandenburg, A. Nordlund, R. F. Stein, U. Torkelsson, ApJ, 446, 741-754 (1995) 
X. Chen, R. E. Taam, ApJ, 412, 254-266 (1993)

X. Chen, M. A. Abramowicz, J.-P. Lasota, R. Narayan, I. Yi, ApJ, 443, L61-L64 (1995)

S. W. Davis, J. M. Stone, Y.-F. Jiang, ApJS, 199, 9, 19pp. (2012)

S. W. Davis, J. M. Stone, M. E. Pessah, ApJ, 713, 52-65 (2010)

J. Dexter, E. Agol, ApJ, 727, L24, 5 pp. (2011)

S. Dibi, S. Drappeau, P. C. Fragile, S. Markoff, J. Dexter, MNRAS, 426, 1928-1939 (2012)

C. Dotan, N. J. Shaviv, MNRAS, 413, 1623-1632 (2011)

A. A. Esin, J. E. McClintock, R. Narayan, ApJ, 489, 865-889 (1997)

J. Ferreira, G. Pelletier, A\&A, 295, 807-832 (1995)

P. C. Fragile, O. M. Blaes, ApJ, 687, 757-766 (2008)

S. Fromang, H. N. Latter, G. Lesur, G. I. Ogilvie, A\&A, submitted (2012), arXiv:1210.6664

S. Fromang, J. Papaloizou, A\&A, 476, 1113-1122 (2007)

S. Fromang, J. Papaloizou, G. Lesur, T. Heinemann, A\&A, 476, 1123-1132 (2007)

A. A. Galeev, R. Rosner, G. S. Vaiana, ApJ, 229, 318-326 (1979)

C. F. Gammie, MNRAS, 297, 929-935 (1998)

C. F. Gammie, ApJ, 522, L57-L60 (1999)

J. Goodman, MNRAS, 339, 937-948 (2003)

F. Haardt, L. Maraschi, ApJ, 380, L51-L54 (1991)

J. F. Hawley, S. A. Balbus, ApJ, 376, 223-233 (1991)

J. F. Hawley, S. A. Balbus, ApJ, 573, 738-748 (2002)

J. F. Hawley, C. F. Gammie, S. A. Balbus, ApJ, 440, 742-763 (1995)

T. Heinemann, J. C. B. Papaloizou, MNRAS, 397, 52-63 (2009)

T. Heinemann, J. C. B. Papaloizou, MNRAS, 397, 64-74 (2009)

S. Hirose, O. Blaes, J. H. Krolik, ApJ, 704, 781-788 (2009)

S. Hirose, J. H. Krolik, O. Blaes, ApJ, 691, 16-31 (2009)

S. Hirose, J. H. Krolik, J. M. Stone, ApJ, 640, 901-917 (2006)

S. Ichimaru, ApJ, 214, 840-855 (1977)

T. Islam, S. Balbus, ApJ, 633, 328-333 (2005)

A. Janiuk, R. Misra, A\&A, 540, A114, 6pp. (2012)

Y.-F. Jiang, J. M. Stone, S. W. Davis, ApJS, 199, 14, 29 pp. (2012)

Y.-F. Jiang, J. M. Stone, S. W. Davis, ApJ, 767, 148, 14 pp. (2013a)

Y.-F. Jiang, J. M. Stone, S. W. Davis, ApJ, submitted (2013b)

S. Kato, M. A. Abramowicz, X. Chen, PASJ, 48, 67-75 (1996)

S. Kato, F. Honma, R. Matsumoto, MNRAS, 231, 37-48 (1988)

S. Kato, T. Yamasaki, M. A. Abramowicz, X. Chen, PASJ, 49, 221-225 (1997)

A. R. King, J. E. Pringle, M. Livio, MNRAS, 376, 1740-1746 (2007)

I. Kotko, J.-P. Lasota, A\&A, 545, A115, 9pp. (2012) (2007)

J. H. Krolik, ApJ, 515, L73-L76 (1999)

J. H. Krolik, S. Hirose, O. Blaes, ApJ, 664, 1045-1056 (2007)

G. Lesur, J. Ferreira, G. I. Ogilvie, A\&A, submitted (2012), arXiv:1210.6660

G. Lesur, P.-Y. Longaretti, MNRAS, 378, 1471-1480 (2007)

J.-P. Lasota, New Astron. Rev., 45, 449-508 (2001)

A. P. Lightman, D. M. Eardley, ApJ, 187, L1-L3 (1974)

D.-B. Lin, W.-M. Gu, T. Liu, M.-Y. Sun, J.-F. Lu, ApJ, 761, 29, 5 pp. (2012)

B. F. Liu, C. Done, R. E. Taam, ApJ, 726, 10, 5 pp. (2011)

D. Lynden-Bell, J. E. Pringle, MNRAS, 168, 603-637 (1974)

M. Machida, K. E. Nakamura, R. Matsumoto, PASJ, 58, 193-202 (2006)

D. L. Meier, Astrophys. Space Sci., 300, 55-65 (2005)

F. Meyer, B. F. Liu, E. Meyer-Hofmeister, A\&A, 354, L67-L70 (2000)

F. Meyer, B. F. Liu, E. Meyer-Hofmeister, A\&A, 361, 175-188 (2000)

F. Meyer, B. F. Liu, E. Meyer-Hofmeister, A\&A, 463, 1-9 (2007)

K. A. Miller, J. M. Stone, ApJ, 534, 398-419 (2000)

N. Murray, J. Chiang, S. A. Grossman, G. M. Voit, ApJ, 451, 498-509 (1995)

R. Narayan, I. V. Igumenshchev, M. A. Abramowicz, ApJ, 539, 798-808 (2000)

R. Narayan, I. V. Igumenshchev, M. A. Abramowicz, PASJ, 55, L69-L72 (2003)

R. Narayan, E. Quataert, I. V. Igumenshchev, M. A. Abramowicz, ApJ, 577, 295-301 (2002)

R. Narayan, A. Sadowski, R. F. Penna, A. K. Kulkarni, MNRAS, 426, 3241-3259 (2012)

R. Narayan, I. Yi, ApJ, 428, L13-L16 (1994)

R. Narayan, I. Yi, ApJ, 444, 231-243 (1995a)

R. Narayan, I. Yi, ApJ, 452, 710-735 (1995b) 
S. C. Noble, J. H. Krolik, J. F. Hawley, ApJ, 711, 959-973 (2010)

G. I. Ogilvie, MNRAS, 306, L9-L13 (1999)

K. Ohsuga, S. Mineshige, ApJ, 736, 2, 18 pp. (2011)

K. Ohsuga, S. Mineshige, M. Mori, Y. Kato, PASJ, 61, L7-L11 (2009)

S. M. O'Neill, C. S. Reynolds, M. C. Miller, K. A. Sorathia, ApJ, 736, 107, 7 pp. (2011)

V. I. Pariev, E. G. Blackman, S. A. Boldyrev, A\&A, 407, 403-421 (2003)

J. Park, C. Ren, E. G. Blackman, X. Kong, Phys. Plasmas, 17, 022901-022901-8 (2010)

R. F. Penna, J. C. McKinney, R. Narayan, A. Tchekhovskoy, R. Shafee, J. E. McClintock, MNRAS, 408, 752-782 (2010)

M. E. Pessah, C.-K. Chan, D. Psaltis, ApJ, 668, L51-L54 (2007)

J. Poutanen, G. Lipunova, S. Fabrika, A. G. Butkevich, P. Abolmasov, MNRAS, 377, 11871194 (2007)

D. Proga, J. M. Stone, T. R. Kallman, ApJ, 543, 686-696 (2000)

E. Quataert, W. Dorland, G. W. Hammett, ApJ, 577, 524-533 (2002)

E. Quataert, A. Gruzinov, ApJ, 539, 809-814 (2000)

M. J. Rees, M. C. Begelman, R. D. Blandford, E. S. Phinney, Nature, 295, 17-21 (1982)

M. A. Riquelme, E. Quataert, P. Sharma, A. Spitkovsky, ApJ, 755, 50, 20 pp. (2012)

A. Różańska, B. Czerny, A\&A, 360, 1170-1186 (2000)

A. Sadowski, R. Narayan, A. Tchekhovskoy, Y. Zhu, MNRAS, in press (2012), arXiv:1212.5050

P. J. Sakimoto, F. V. Coroniti, ApJ, 247, 19-31 (1981)

N. I. Shakura, R. A. Sunyaev, A\&A, 24, 337-355 (1973)

N. I. Shakura, R. A. Sunyaev, MNRAS, 175, 613-632 (1976)

S. L. Shapiro, A. P. Lightman, D. M. Eardley, ApJ, 204, 187-199 (1976)

P. Sharma, G. W. Hammett, E. Quataert, ApJ, 596, 1121-1130 (2003)

P. Sharma, G. W. Hammett, E. Quataert, J. M. Stone, ApJ, 637, 952-967 (2006)

P. Sharma, E. Quataert, G. W. Hammett, J. M. Stone, ApJ, 667, 714-723 (2007)

J. Shi, J. H. Krolik, S. Hirose, ApJ, 708, 1716-1727 (2010)

N. Shibazaki, R. Hōshi, Prog. Theor. Phys., 54, 706-718 (1975)

J. B. Simon, K. Beckwith, P. J. Armitage, MNRAS, 422, 2685-2700 (2012)

J. B. Simon, J. F. Hawley, ApJ, 707, 833-843 (2009)

J. Smak, PASP, 96, 5-18 (1984)

A. Socrates, S. W. Davis, O. Blaes, ApJ, 601, 405-413 (2004)

A. Socrates, ApJ, 719, 784-789 (2010)

K. A. Sorathia, C. S. Reynolds, P. J. Armitage, ApJ, 712, 1241-1247 (2010)

J. M. Stone, J. F. Hawley, C. F. Gammie, S. A. Balbus, ApJ, 463, 656-673 (1996)

T. K. Suzuki, S.-I. Inutsuka, ApJ, 691, L49-L54 (2009)

R. Svensson, A. A. Zdziarski, ApJ, 436, 599-606 (1994)

R. E. Taam, D. N. C. Lin, ApJ, 287, 761-768 (1984)

H. R. Takahashi, K. Ohsuga, Y. Sekiguchi, T. Inoue, K. Tomida, ApJ, in press (2012), arXiv: 1212.4910

N. J. Turner, ApJ, 605, L45-L48 (2004)

N. J. Turner, O. M. Blaes, A. Socrates, M. C. Begelman, S. W. Davis, ApJ, 624, 267-288 (2005)

N. J. Turner, J. M. Stone, J. H. Krolik, T. Sano, ApJ, 593, 992-1006 (2003)

X.-B. Wu, MNRAS, 292, 113-119 (1997)

X.-B. Wu, Q.-B. Li, ApJ, 469, 776-783 (1996)

T. Yamasaki PASJ, 49, 227-233 (1997)

F. Yuan, MNRAS, 324, 119-127 (2001)

F. Yuan, ApJ, 594, L99-L102 (2003) 